\title{
Ansätze zur Darstellung nicht- und schwach idiomatischer verbonominaler Wortverbindungen in der zweisprachigen (Lerner-)Lexikografie Deutsch-Finnisch (Beschreibung eines Forschungsvorhabens)
}

\author{
Antje Heine (Leipzig/Helsinki)
}

\begin{abstract}
The study focuses on verb-noun-connections of the type verb + subject (accusative) and verb + preposition + subject. They will be described systematically, among themselves and in relation to free word connections. The units to be examined will be taken from electronic corpora. They will be analysed with regard to their utilisation. The second part of the research project focuses on the elaboration of Finnish analogies to these units, asking, whether the results can be used for bilingual lexicography (German-Finnish).
\end{abstract}

\section{$1 \quad$ Untersuchungsgegenstand}

Phraseologismen, d.h. mehr oder weniger feste Wortverbindungen stellen für NichtMuttersprachler besonders häufige Fehlerquellen dar. Beginnend mit dem Prozess des Spracherwerbs und sich fortsetzend bis zur (professionellen) Übersetzung steht man immer wieder vor Problemen, die von grammatisch-strukturellen Fragen über die Bedeutung bis hin zur Anwendung reichen, also gleichermaßen auf den Ebenen Morphosyntax, Semantik und Pragmatik anzusiedeln sind.

Aus dem weiten Gegenstandsbereich der Phraseologie sollen in dem Forschungsprojekt ${ }^{1}$ Verb-Nomen-Verbindungen des Typs Verb $+\operatorname{Substantiv}_{\text {Akkusativ }}\left(\mathrm{V}+\mathrm{S}_{\mathrm{a}}\right)$ und Verb + Präposition + Substantiv $(\mathrm{V}+\mathrm{pS})$ herausgegriffen werden. Sie sind anzusehen als ein Kontinuum von nicht-idiomatischen Kollokationen (Fenster putzen; in Strömen regnen) über nicht- bzw. schwach idiomatische Funktionsverbgefüge (Anwendung finden; in Kontakt treten) bis hin zu teil- oder vollidiomatischen Wortidiomen (sein Herz an jmdn verlieren; jmdn auf die Folter spannen) und weiteren, noch zu ermittelnden Gruppen / komplexen Prädikaten (wie Maschine schreiben; Eis laufen). Während die oben festgelegten Strukturtypen $\mathrm{V}+\mathrm{S}_{\mathrm{a}}$ und $\mathrm{V}+\mathrm{pS}$ für die letztgenannten Gruppen wie auch für

\footnotetext{
${ }^{1}$ Das Projekt wird am Germanistischen Institut der Universität Helsinki durchgeführt und zunächst für ein Jahr in Form eines Post-Doc-Stipendiums von der Alexander von Humboldt-Stiftung sowie der Universität Helsinki finanziert.
} 
Funktionsverbgefüge den Normalfall darstellen, sind sie für Wortidiome und Kollokationen als eine Vorkommensweise unter anderen zu betrachten.

Arbeiten im Bereich der Phraseologie werden durch den Umstand erschwert, dass es immer noch an einer einheitlichen Subklassifizierung und erst recht an einer ausreichenden und allgemein anerkannten Beschreibung des phraseologischen Inventars fehlt. In diesem Zusammenhang ist in jedem Falle Korhonen (2002: 406ff.) zuzustimmen, der einerseits die in der neueren Forschungsliteratur vertretene Ansicht teilt, "vor der Überbewertung von Klassifikationsversuchen bzw. vor starren Ordnungsschemata" sei zu warnen, andererseits aber auch betont, dass für "bestimmte Anwendungsmöglichkeiten" solche Klassifikationsversuche unabdingbar sind. Daher wird es auch im Rahmen des Forschungsprojektes, das primär an phraseografischen (und damit verbunden auch phraseodidaktischen) Zielen ausgerichtet ist, notwendig sein, die ausgewählten Einheiten systematisch zu beschreiben und für deren Subklassifizierung entsprechende Ein- und Zuordnungskriterien zu erarbeiten. Für die Funktionsverbgefüge liegen diese aus einer früheren Untersuchung im Wesentlichen bereits vor, für Kollokationen gilt es, die mittlerweile recht umfassende Literatur (cf. unter anderem Burger 2003; Hollós 2004; Steyer 2004; Wotjak/Heine 2005) auszuwerten und schließlich entsprechende Kriterien festzulegen. Bei den weiteren mehr oder weniger festen, möglicherweise als komplexe Prädikate anzusehenden Verb-Substantiv-Verbindungen handelt es sich mehrheitlich um Inkorporierungen; deren systematische Beschreibung ist allerdings nicht ganz unproblematisch, da ein Teil von ihnen erst mit der - immer noch nicht endgültig abgeschlossenen - Rechtschreibreform den Phraseologismen zuzuordnen ist, während es sich zuvor um Einwortlexeme handelte (cf. Suchsland 1999).

Auf Grund der bereits in großem Umfang vorliegenden Forschungsergebnisse zu den Wortidiomen, zu denen insbesondere Korhonen einen entscheidenden Beitrag geleistet hat und die eine praktische Umsetzung unter anderem in Form seines Idiomwörterbuchs "Alles im Griff. Homma hanskassa" (Korhonen 2001b) erfahren haben, sollen diese Wortverbindungen in meinem Forschungsprojekt weitgehend außer Betracht bleiben bzw. lediglich dort herangezogen werden, wo eine Kontrastierung zu den anderen genannten Subklassen notwendig erscheint.

Parallel zur Arbeit am Deutschen gilt es zu erfassen, inwieweit diese Überlegungen auch für das finnische Sprachsystem zutreffend sind bzw. welche Unterschiede auf Grund der verschiedenen sprachtypologischen Gruppen, denen beide Sprachen angehören, von vornherein erwartbar sind. ${ }^{2}$ Beispielsweise gibt es im Finnischen nur wenige Präpositionen die ja bei den ausgewählten Strukturtypen eine bedeutende Rolle spielen - anstelle dessen eine Reihe von Postpositionen.

2 Dabei stellen die bereits erwähnten umfassenden Vorarbeiten von Korhonen bzw. überhaupt am Germanistischen Institut der Universität Helsinki eine wichtige Basis dar. 


\section{Möglichkeiten der Ermittlung mehr oder weniger fester verbonominaler Wortverbindungen und deren Gebrauchspräferenzen}

Die Beschreibung der einzelnen Klassen von Verb-Substantiv-Verbindungen geht mit zwei weiteren Aspekten einher: Zum einen erfordert sie eine Abgrenzung von freien Wortkombinationen, zum anderen wirft sie die Frage auf, auf welche Weise sich derartige Wortverbindungen ermitteln lassen und wie sich ihr Vorkommen in der Sprachverwendung gestaltet. Für beide Fragestellungen muss auf das Potential elektronischer Korpora verwiesen werden; die Analyse elektronisch aufbereiteter Massendaten bietet die Möglichkeit Üblichkeiten, Präferenzen und Restriktionen im Sprachgebrauch zu ermitteln, die rein introspektiv in dieser Breite nicht zu eruieren sind. So erlaubt es beispielsweise die so genannte Kookkurrenzanalyse ${ }^{3}$ (cf. Steyer 2002) von COSMAS II (http://www.idsmannheim.de/cosmas2/), den Wahrscheinlichkeitsgrad des Miteinandervorkommens zweier (oder mehrerer) lexikalischer Einheiten zu ermitteln. Ein entsprechend hoher Kohäsionsgrad verweist wiederum auf potentielle neue lexikalische Einheiten und damit Phraseologismen. D. h., mit Hilfe ausreichend großer und in ihrer Zusammensetzung brauchbarer Korpora können zunächst usuelle Wortverbindungen extrahiert und in einem zweiten Schritt klassifiziert werden, wenngleich die automatisierte Arbeit des Computers lediglich als Ergänzung zur (interpretativen) wissenschaftlichen Arbeit des Linguisten anzusehen ist (cf. auch Teubert 1999: 295). Über die Identifikation lexikalisierter Verb-Nomen-Verbindungen hinaus wird mittels Korpusanalyse der tatsächliche Sprachgebrauch sichtbar, indem einerseits bestimmte Präferenzen, andererseits aber auch weniger übliche und doch Akzeptabilität aufweisende Formen herausgefiltert und damit letztlich auch Restriktionen ermittelt werden können.

\section{$3 \quad$ Vorarbeiten für die (lerner-)lexikografische praktische Umsetzung}

In den letzten Jahren ist wiederholt und nicht nur für das Deutsche auf einen Mangel an einund zweisprachigen phraseologischen Wörterbüchern bzw. auf eine unbefriedigende Darstellung von Phraseologismen in diesen wie auch in allgemeinen Wörterbüchern aufmerksam gemacht worden (cf. u. a. die Beiträge in Barz/Schröder 1996 (insbesondere Wotjak/Dobrovol'skij) sowie Wiegand 1998 und 2002; Korhonen 2004; Heine 2004). Bei der Durchsicht dieser evaluierenden Arbeiten treten zwei Aspekte besonders deutlich hervor: zum einen die oben erwähnte Tatsache, dass trotz des momentan überaus großen Interesses an Phraseologie und Lexikografie, insbesondere der Phraseografie, die Diskussionen der Linguisten über das phraseologische Inventar und dessen Subklassifizierung bislang zu keinen gesicherten oder wenigstens mehrheitlich anerkannten Ergebnissen geführt haben, zum anderen aber auch der Umstand, dass die durchaus vorhandenen Erkenntnisse und Ergebnisse der Phraseologen bei der Er- und Überarbeitung von Wörterbüchern z. T. nicht ausreichend Berücksichtigung fanden. So treten auch immer mal wieder "neue Ideen" hervor, zu denen ich

\footnotetext{
${ }^{3}$ Ich beziehe mich im Folgenden auf die am Institut für Deutsche Sprache Mannheim und damit auch in dessen Korpus COSMAS II, auf dem die beschriebene Analyse basiert, verwendeten Termini.
} 
den Versuch van Pottelberges (2001) zähle, die Funktionsverbgefüge als eine eigenständige Kategorie mehr als nur in Frage zu stellen, aber auch die Ansicht Hausmanns (2004), dass Wortidiome wie Bauklötze staunen gleichzeitig Kollokationen darstellen.

So ist es weiterhin nötig, einer phraseologischen Arbeit eine (eigene) Definition des Untersuchungsgegenstandes voranzustellen, da es mit Blick auf die Wörterbuchpraxis, insbesondere für die Erstellung von ein- und zweisprachigen Lernerwörterbüchern, klarer Zuordnungskriterien als Voraussetzung für eine nachvollziehbare Artikelgestaltung bedarf. Ich gehe mit denjenigen Linguisten (cf. z. B. Wiegand 2000) konform, die trotz der oben angesprochenen Schwierigkeiten gesonderte, klar unterscheidbare Suchzonen für die einzelnen phraseologischen Bereiche (bspw. für Wortidiome, Funktionsverbgefüge, Kollokationen, kommunikative Formeln und gegebenenfalls auch Sprichwörter) für notwendig erachten. Dies lässt sich nicht zuletzt damit begründen, dass - betrachtet man allein die hier den Untersuchungsgegenstand bildenden verbonominalen Verbindungen - die einzelnen Untergruppen einen unterschiedlichen Grad an Analysierbarkeit und Asynthetisierbarkeit ${ }^{4}$ aufweisen. Daraus folgt aus sprachproduktiver Perspektive, dass sie auch in unterschiedlichem Grade morphosyntaktischen, semantischen und pragmatischen Restriktionen (bspw. hinsichtlich ihrer Kompatibilität oder in ihrer Situationsspezifik) unterliegen und - mit Blick auf die Sprachrezeption - eine mehr oder weniger gute Verständlichkeit bzw. Durchschaubarkeit aufweisen.

Diese hier dargelegten Defizite in der bisherigen lexikografischen Praxis lassen sich, zumindest zu einem großen Teil (und zunächst auf die Funktionsverbgefüge beschränkt), mit Hilfe der Korpusanalyse und dem im Folgenden vorgestellten Raster reduzieren. Dieses Raster dient dazu, die ausgewählten lexikalischen Einheiten nach ihrer Ermittlung und im Vorfeld ihrer konkreten "Verarbeitung" für Wörterbuchzwecke einen mehrstufigen Beschreibungsalgorithmus durchlaufen zu lassen, der die folgenden Untersuchungsaspekte in sich vereint: a) die Nennformschreibung (als Ausgangspunkt für eine alphabetische Zugriffsstruktur im Wörterbuch); b) die morphosyntaktische Ausdrucksstruktur mit Hinweisen auf die morphosyntaktische Beschaffenheit der Umgebungen (Aktanten) sowie auf Gebrauchsrestriktionen; c) die Inhaltsstruktur mit Hinweisen auf eine bestimmte semantische Klassenzugehörigkeit sowie die Einordnung in ein lexikalisch-semantisches Feld (als Ausgangspunkt für eine onomasiologisch orientierte Zugriffsstruktur im Wörterbuch) und auf die semantische Beschaffenheit der Umgebungen; d) Synonyme oder andere Paraphrasierungsmöglichkeiten; e) Hinweise zur Stilebene, zu Sprechereinstellungen, zur zeitlichen und räumlichen Einordnung, zu Textsortenspezifika etc. und f) Korpusbelege für a) bis e), die die Funktion erfüllen, die morphosyntaktischen, semantischen und pragmatischen Erläuterungen prototypisch zu unterstützen (cf. Neubauer 1998). Dies sei anhand der folgenden Tabelle demonstriert:

\footnotetext{
${ }^{4}$ Nach Lehr (1998: 258, in Anlehnung an Bahns, Hausmann u. a.) sind sprachliche Einheiten asynthetisierbar, wenn sie "nicht unter gleichzeitigem Ausschluß anderer fehlerhafter oder ungebräuchlicher Wortkombinationen, deren Elemente mit den ihren nach syntaktischen und semantischen Regeln synonym sind, gebildet werden" können, und analysierbar, wenn "ihre Gesamtbedeutung [...] unter Berücksichtigung der syntaktischen Regeln aus den Bedeutungen der einzelnen Komponenten erschlossen werden" kann.
} 
Antje Heine: Ansätze zur Darstellung nicht- und schwach idiomatischer verbonominaler

Wortverbindungen in der zweisprachigen (Lerner-)Lexikografie Deutsch-Finnisch

\begin{tabular}{|c|c|c|}
\hline Ebene & Untersuchungsaspekt & Mögliche Realisierungen \\
\hline \multirow{4}{*}{ Morphosyntax } & Artikelwörter & $\begin{array}{l}\text { - bestimmter Artikel } \\
\text { - unbestimmter Artikel } \\
\text { - Nullartikel } \\
\text { - adjektivisches Demonstrativpronomen } \\
\text { - adjektivisches Possessivpronomen }\end{array}$ \\
\hline & Adjektivattribut & $\begin{array}{l}\text { - obligatorische Erweiterung } \\
\text { - fakultative Erweiterung }\end{array}$ \\
\hline & Aktanten & $\begin{array}{l}\text { - obligatorische Aktanten } \\
\text { - fakultative Aktanten }\end{array}$ \\
\hline & morphosyntaktische Üblichkeiten & $\begin{array}{l}\text { - häufig mit Negation } \\
\text { - häufig mit Modalverben } \\
\text { - häufig in bestimmten Zeitformen } \\
\text { etc. }\end{array}$ \\
\hline \multirow{5}{*}{ Semantik } & $\begin{array}{l}\text { Einordnung in ein lexikalisch- } \\
\text { semantisches Feld }\end{array}$ & $\begin{array}{l}\text { - Feld der allgemeinen Existenz } \\
\text { - Feld der speziellen Existenz } \\
\text { - Feld der Differenz } \\
\text { - Feld der Relation und des geistigen Handelns } \\
\text { etc. }\end{array}$ \\
\hline & Semantische Merkmale des FVG & $\begin{array}{l}\text { - grenzbezogen } \\
\text { - nicht-grenzbezogen } \\
\text { - kausativ } \\
\text { - passiv }\end{array}$ \\
\hline & $\begin{array}{l}\text { Semantische Merkmale der } \\
\text { Umgebung: Subjekt-/ } \\
\text { Objektklassen }^{5}\end{array}$ & $\begin{array}{l}\text { - Lebewesen (Personen, Tiere etc.) } \\
\text { - Institutionen } \\
\text { - Stoffe } \\
\text { - Gegenstände } \\
\text { etc. }\end{array}$ \\
\hline & $\begin{array}{l}\text { Semantische Merkmale der } \\
\text { Umgebung: Adjektivattribut (des } \\
\text { Nominalteils) }\end{array}$ & $\begin{array}{l}\text { - Einschränkungen in der Kompatibilität (räumliche, } \\
\text { bewertende, Intensität ausdrückende etc. Adjektive) }\end{array}$ \\
\hline & Paraphrasierbarkeit & $\begin{array}{l}\text { - stammverwandtes Vollverb (mit/ohne Präfix) } \\
\text { - stammverwandtes Adjektiv + Kopulaverb } \\
\text { - andere Paraphrase }\end{array}$ \\
\hline \multirow{4}{*}{ Pragmatik } & Diaphasische Markiertheit & $\begin{array}{l}\text { - Zeitbezug: veraltet, veraltend etc. } \\
\text { - fachsprachliche Markiertheit: Jura, Biologie, } \\
\text { Amtssprache etc. } \\
\text { - situative Markiertheit: Redeanlass, Ort, Sender/ } \\
\text { Empfänger etc. } \\
\text { - Stil: gehoben }\end{array}$ \\
\hline & Diastratische Markiertheit & - Schicht-, Geschlechts-, Alters-, Gruppenspezifik \\
\hline & Diamesische Markiertheit & $\begin{array}{l}\text { - gesprochen } \\
\text { - geschrieben }\end{array}$ \\
\hline & Diatopische Markiertheit & $\begin{array}{l}\text { - dialektale Varianten: ostdeutsch, süddeutsch etc. } \\
\text { (-nationale Varianten: österreichisch, schweizerhoch- } \\
\text { deutsch etc.) }\end{array}$ \\
\hline
\end{tabular}

\footnotetext{
${ }^{5}$ Diese Angaben beziehen sich immer auf den Gebrauch des Funktionsverbgefüges im Aktivsatz.
} 
Aufgabe der zweiten Phase des Forschungsprojektes wird es folglich sein, mit Hilfe der Analyse elektronischer Korpora die für die Lemmaselektion und Mikrostruktur eines zweisprachigen Wörterbuches notwendigen Informationen zu extrahieren, interpretieren und systematisieren.

\section{Kontrastive Analyse Deutsch-Finnisch}

Parallel dazu wird am unilateralen Sprachvergleich Deutsch-Finnisch zu arbeiten sein. Auf der Grundlage des vorgestellten Beschreibungsrasters sind mit Hilfe finnischer Korpora sowie Informantenbefragungen von Muttersprachlern die finnischen Äquivalente für die ausgewählten Phraseologismen zu ermitteln. Nach Korhonen/Wotjak (2001: 227) ist dabei grob - von folgenden Äquivalenztypen auszugehen, die einer weiteren Spezifizierung und v. a. Konkretisierung für das Sprachenpaar Deutsch-Finnisch bedürfen: a) Es existiert ein äquivalenter Phraseologismus, der im Vergleich zur deutschen lexikalischen Einheit Kongruenz, Teilkongruenz oder eine gänzlich andere Struktur aufweist, oder b) das Finnische verfügt nicht über ein phraseologisches Äquivalent, d.h., die deutsche verbonominale Verbindung kann im Finnischen mit einer Wortbildungskonstruktion, einem primären Einzellexem oder einer freien Formativkette wiedergegeben werden. ${ }^{6}$ Besonders wichtig, sowohl aus fremdsprachendidaktischer Perspektive als auch für die Übersetzung, ist es, auch kleinste voneinander abweichende Bedeutungsnuancen sowie Unterschiede in der Verbindbarkeit wie auch auf pragmatischer Ebene zu erfassen. Letzteres erweist sich sicherlich als besondere Herausforderung, da es einer gewissen Korpusgröße und -zusammensetzung bzw. einer entsprechenden Anzahl muttersprachlicher Informanten bedarf um diesbezüglich zu repräsentativen Ergebnissen zu gelangen.

\section{$5 \quad$ Zusammenfassung und Ausblick}

Ein erstes Ziel sollte erreicht sein, wenn eine Liste $^{7}$ unterschiedlich strukturierter und auf der Basis der bereits existierenden Vorarbeiten sowie der weiteren theoretischen Überlegungen in der ersten Phase des Projektes möglichst klar subklassifizierter Verb-Nomen-Verbindungen erstellt ist, die Funktionsverbgefüge unter ihnen zunächst für das Deutsche entsprechend des Mehrebenenmodells beschrieben und ihre finnischen Entsprechungen ermittelt sind. Als weiteres Forschungsvorhaben bleibt die Ausweitung der zu untersuchenden Einheiten auf Kollokationen und weitere nicht- und schwach idiomatische Verb-Nomen-Verbindungen sowie - längerfristig - die konkrete Umsetzung der Ergebnisse in Gestalt eines Lexikons verbonominaler Einheiten. Ein solches Nachschlagewerk wäre vor allem für den Fremdsprachenerwerb Deutsch wie auch für die Übersetzungswissenschaft von großem Nutzen und wird von beiden Seiten auch nachdrücklich als Desiderat betrachtet. Zudem könnten die Untersuchungsergebnisse in zukünftigen allgemeinsprachigen Wörterbüchern

\footnotetext{
${ }^{6}$ Im Gegensatz dazu geht Farø (2004) von einem sechsstufiges Konvergenz-Modell aus.

${ }^{7}$ Die nicht unerhebliche Frage nach dem Umfang einer solchen Liste lässt sich an dieser Stelle allerdings noch nicht klären, da sie von verschiedensten Faktoren (auch vor Ort) beeinflusst sein wird.
} 
Widerspiegelung finden. Denkbar wäre ebenso eine Ausweitung der Untersuchung, verbunden mit einer Adaption des Beschreibungsmodells, auf weitere Sprachen.

\section{Literaturangaben}

Bahns, Jens (1993): "Wer eine günstige Gelegenheit verpaßt, kann beträchtlichen Schaden davontragen. Kollokationen in Langenscheidts Großwörterbuch Deutsch als Fremdsprache". Lernen in Deutschland 2: 137-155.

Barz, Irmhild/Schröder, Marianne (eds.) (1996): Das Lernerwörterbuch Deutsch als Fremdsprache in der Diskussion. Heidelberg.

Barz, Irmhild/Bergenholtz, Henning/Korhonen, Jarmo (eds.) (2005): Schreiben, Verstehen, Übersetzen, Lernen. Zu ein- und zweisprachigen Wörterbüchern mit Deutsch. Frankfurt a.M. (= Finnische Beiträge zur Germanistik 14).

Burger, Harald (2003): Phraseologie. Eine Einführung am Beispiel des Deutschen. Berlin.

Cheon, Mi-Ae (1998): Zur Konzeption eines phraseologischen Wörterbuchs für den Fremdsprachler. Tübingen. (= Lexicographica Series Maior 89).

Farø, Ken (2004): "Am Anfang. Zur Erforschung der dänisch-deutschen Idiomatik". In: Földes, Csaba/Wirrer, Jan (eds.): Phraseologismen als Gegenstand sprach- und kulturwissenschaftlicher Forschung. Akten der Europäischen Gesellschaft für Phraseologie und des Westfälischen Arbeitskreises "Phraseologie/Parömiologie" (Loccum 2002). Baltmannsweiler. (= Phraseologie und Parömiologie 15): 105-117.

Götz, Dieter/Haensch, Günther/Wellmann, Hans ( $\left.{ }^{3} 2003\right)$ : Langenscheidts Großwörterbuch Deutsch als Fremdsprache. Berlin etc.

Hausmann, Franz Josef (1985): "Kollokationen im deutschen Wörterbuch". In: Bergenholtz, Henning/Mugdan, Joachim (eds.): Lexikographie und Grammatik. Akten des Essener Kolloquiums zur Grammatik im Wörterbuch 28.-30.6.1984. Tübingen. (= Lexicographica Series Maior 3): 118-129.

Hausmann, Franz Josef (2004): "Was sind eigentlich Kollokationen?". In: Steyer, Kathrin (ed.): Wortverbindungen - mehr oder weniger fest. Institut für Deutsche Sprache Jahrbuch 2003. Berlin/New York: 309-334.

Heine, Antje (2004): "Zur Darstellung von Funktionsverbgefügen in Langenscheidts Großwörterbuch Deutsch als Fremdsprache". In: Földes, Csaba/Wirrer, Jan (eds.): Phraseologismen als Gegenstand sprach- und kulturwissenschaftlicher Forschung. Akten der Europäischen Gesellschaft für Phraseologie und des Westfälischen Arbeitskreises "Phraseologie/Parömiologie" (Loccum 2002). Baltmannsweiler. (= Phraseologie und Parömiologie 15): 351-364.

Helbig, Gerhard/Buscha, Joachim $\left({ }^{19} 2001\right)$ : Deutsche Grammatik - Ein Handbuch für den Ausländerunterricht. Leipzig etc.

Hollós, Zita (2004): Lernerlexikographie: Syntagmatisch. Konzeption für ein deutschungarisches Lernerwörterbuch. Tübingen. (= Lexicographica Series Maior 116).

Kempcke, Günter (ed.) (2000): Wörterbuch Deutsch als Fremdsprache. Berlin/New York. 
Korhonen, Jarmo (ed.) (2001a): Von der mono- zur bilingualen Lexikografie für das Deutsche. Frankfurt/M. (= Finnische Beiträge zur Germanistik 6).

Korhonen, Jarmo (2001b): Alles im Griff. Homma hanskassa. Saksa-suomi-idiomisanakirja. Idiomwörterbuch Deutsch-Finnisch. Helsinki.

Korhonen, Jarmo (2002): "Typologien der Phraseologismen: Ein Überblick". In: Cruse, D. Alan et. al. (eds.): Lexikologie. Lexicology. Ein internationales Handbuch zur Natur und Struktur von Wörtern und Wortschätzen. Berlin/New York. (= HSK 21,1): 402-407.

Korhonen, Jarmo (2004): "Duden 11 - Nutzungsverfahren aus der DaF-Perspektive". In: Steyer, Kathrin (ed.): Wortverbindungen - mehr oder weniger fest. Institut für Deutsche Sprache Jahrbuch 2003. Berlin/New York: 360-393.

Korhonen, Jarmo/Wotjak, Barbara (2001): "Kontrastivität in der Phraseologie". In: Helbig, Gerhard et. al. (eds.): Deutsch als Fremdsprache. Ein internationales Handbuch. 1. Halbband. Berlin/New York. (= HSK 19, 1): 224-235.

Kotschi, Thomas (1998): "Charge. Zwei Einträge aus dem Wörterbuch französischer Funktionsverbgefüge". In: Figge, Udo/Klein, Franz-Josef/Martinez-Moreno, Annette (eds.): Grammatische Strukturen und grammatischer Wandel im Französischen. Festschrift für Klaus Hunnius. Bonn. (= Abhandlungen zur Sprache und Literatur 117): 309-333.

Lehr, Andrea (1998): "Kollokationen in Langenscheidts Großwörterbuch Deutsch als Fremdsprache". In: Wiegand, Herbert Ernst (ed.): Perspektiven der pädagogischen Lexikographie des Deutschen. Untersuchungen anhand von "Langenscheidts Großwörterbuch Deutsch als Fremdsprache". Tübingen. (= Lexicographica Series Maior 86): 256-281.

Neubauer, Fritz (1998): "Kompetenzbeispiele in Langenscheidts Großwörterbuch Deutsch als Fremdsprache". In: Wiegand, Herbert Ernst (ed.): Perspektiven der pädagogischen Lexikographie des Deutschen. Untersuchungen anhand von "Langenscheidts Großwörterbuch Deutsch als Fremdsprache". Tübingen. (= Lexicographica Series Maior 86): 247-255.

Pottelberge, Jeroen van (2001): Verbonominale Konstruktionen, Funktionsverbgefüge: Vom Sinn und Unsinn eines Untersuchungsgegenstandes. Heidelberg.

Steyer, Kathrin (2000): "Usuelle Wortverbindungen des Deutschen. Linguistisches Konzept und lexikografische Möglichkeiten". Zeitschrift Deutsche Sprache 2: 101-125.

Steyer, Kathrin (2002): "Wenn der Schwanz mit dem Hund wedelt. Zum linguistischen Erklärungspotenzial der korpusbasierten Kookkurrenzanalyse". In: Haß-Zumkehr, Ulrike/Kallmeyer, Werner/Zifonun, Gisela (eds.): Ansichten der deutschen Sprache. Festschrift für Gerhard Stickel zum 65. Geburtstag. Tübingen: 215-236.

Steyer, Kathrin (ed.) (2004): Wortverbindungen - mehr oder weniger fest. Institut für Deutsche Sprache Jahrbuch 2003. Berlin/New York.

Suchsland, Peter (1999): "Soll man Kopf stehend und freudestrahlend Eis laufen? Linguistische Fußangeln der neuen Rechtschreibung". In: Skibitzki, Bernd/Wotjak, Barbara (eds.): Linguistik und Deutsch als Fremdsprache. Festschrift für Gerhard Helbig zum 70. Geburtstag. Tübingen: 209-226. 
Teubert, Wolfgang (1999): "Korpuslinguistik und Lexikografie". Deutsche Sprache 4: 292-313.

Wiegand, Herbert Ernst (ed.) (1998): Perspektiven der pädagogischen Lexikographie des Deutschen. Untersuchungen anhand von "Langenscheidts Großwörterbuch Deutsch als Fremdsprache". Tübingen. (= Lexicographica Series Maior 86).

Wiegand, Herbert Ernst (2000): "Über Suchbereiche, Suchzonen und ihre textuellen Strukturen in Printwörterbüchern. Ein Beitrag zur Theorie der Wörterbuchform". In: Wiegand, Herbert Ernst (ed.): Wörterbücher in der Diskussion IV. Tübingen. (= Lexicographica Series Maior 100): 233-301.

Wiegand, Herbert Ernst (ed.) (2002): Perspektiven der pädagogischen Lexikographie des Deutschen II. Untersuchungen anhand von "Langenscheidts Großwörterbuch Deutsch als Fremdsprache". Tübingen. (= Lexicographica Series Maior 110).

Wotjak, Barbara/Dobrovol'skij, Dmitrij (1996): "Phraseologismen im Lernerwörterbuch". In: Barz, Irmhild/Schröder, Marianne (eds.): Das Lernerwörterbuch Deutsch als Fremdsprache in der Diskussion. Heidelberg: 243-264.

Wotjak, Barbara/Heine, Antje (2005): "Überlegungen zur Abgrenzung und Beschreibung verbonominaler Wortverbindungen (Wortidiome, Funktionsverbgefüge, Kollokationen): Vorleistungen für die (lerner-)lexikographische Praxis". Deutsch als Fremdsprache 3: 143-153. 